\title{
Interactions between Dorsal Root Axons and Their Target Motor Neurons in Developing Mammalian Spinal Cord
}

\author{
William D. Snider, Li Zhang, Saira Yusoof, Narender Gorukanti, and Chock Tsering \\ Department of Neurology and Neurological Surgery (Neurology), Washington University Medical School, St. Louis, \\ Missouri 63110
}

\begin{abstract}
We have utilized the lipid-soluble tracers Dil and DiA to investigate interactions between group la dorsal root afferent axons and their target motor neurons in developing rat spinal cord. We show here that la axons project toward motor pools in fascicles that exhibit a considerable degree of spatial order. A rough topography is present in that axons that innervate medially located axial motor neurons cross over others in the intermediate zone and follow a separate path along the midline toward their appropriate targets. Surprisingly, we have also found that motor neuron dendritic projections are well established in the transverse plane prior to the arrival of la afferents. Although dendrites from motor pools innervating limb muscles project directly in the path of incoming la afferents, they do not guide afferents to appropriate motor pools. The la afferents pass over the distal dendrites and grow all the way to the border between gray and developing white matter. A significant amount of terminal branching and bouton formation is in the vicinity of motor neuron somata and proximal regions of the dendritic arbors. Few boutons are found near dendrites that project dorsal to the motor pools, and virtually no boutons are found on dendrites in white matter.
\end{abstract}

Our results show that la afferent axons are not guided to appropriate motor pools by random encounters with motor dendrites, and raise the possibility that mechanisms exist that promote an orderly projection of la afferents to particular regions of the ventral horn. The striking lack of innervation of white matter and dorsally directed dendrites by la afferents raises the question of whether descending and intersegmental systems have their initial interactions with these regions of the motor neuron dendritic arbor.

The monosynaptic connection between group Ia dorsal root afferent axons and motor neurons in the spinal cord is a favored circuit for the study of how specific connections are formed in the CNS because of the ready accessibility of its afferent and efferent components. In frog and chick, it has been demonstrated that highly specific connections between afferents from a paricular muscle and motor neurons projecting to that muscle are

Received Dec. 9, 1991; revised Mar. 18, 1992; accepted Apr. 14, 1992.

We thank J. Lichtman, R. Wilkenson, P. VanMier, and A. Burkhalter for critical comments on the manuscript. This work was supported by NIH Grant NS 25936 and NSF Grant BNS-91-12910 to W.D.S. and NIH Program Project NS 17763.

Correspondence should be addressed to William D. Snider, M.D., Department of Neurology, Box 8111, Washington University School of Medicine, 660 South Euclid Avenue, St. Louis, MO 63110.

Copyright (C) 1992 Society for Neuroscience $0270-6474 / 92 / 123494-15 \$ 05.00 / 0$ formed at an early developmental stage (Frank and Westerfield, 1983; Lee and O'Donovan, 1991). These connections appear to be specific from the earliest time at which synaptic potentials can be recorded and do not undergo rearrangement as development proceeds (Frank and Westerfield, 1983; Lee and O'Donovan, 1991).

The manner in which these specific sensory-motor neuron conncctions arc cstablished has been characterized anatomically in frog, where bulk labeling with HRP fully labels axonal and dendritic arbors and where intracellular and intraxonal staining is feasible even during development. These studies have shown that afferents arrive in the region of contact first and that motor neuron dendrites grow into the area occupied by afferent axons (Jackson and Frank, 1987). Interestingly, muscle afferents in frog do not appear to grow along a particular pathway, and spatial order in the projection of these afferents has not been described (Jackson and Frank, 1987; see also Lichtman et al., 1984). In chicks and mammals, studies that have employed bulk labeling of dorsal and ventral roots with HRP have revealed that Ia afferents and motor dendrites project almost simultaneously into a dense area of interaction at the same developmental stage at which connections can be demonstrated physiologically (Smith, 1983; Kudo and Yamada, 1987; Lee et al., 1988; Davis et al., 1989; Ziskind-Conhaim, 1990). Although the time course of the interaction has been established in this way, labeling with HPR becomes increasingly granular and less satisfactory, particularly for dendrites as development proceeds in these species. Furthermore, since axons and dendrites are both labeled brown, it is difficult to tell one from the other in areas where the neuropil is dense. Therefore, a detailed view of interactions between afferents and dendrites has not been achieved even in this simple system in higher vertebrates.

The recent introduction of intensely fluorescent lipid-soluble tracers provides a convenient technique for studying both afferent and efferent components of circuits in the developing CNS, particularly in mammals where other methods may be difficult to utilize (Honig and Hume, 1986; Godement et al., 1987; Snider and Palavali, 1990). A major advantage over HRP is that these dyes are available in different chromophores (Chua et al., 1990). Thus, interactions between innervating axons and dendrites can be studied because axons can be distinguished from dendrites even in areas where the neuropil is dense. In the present study, we have utilized lipid-soluble tracers during rat embryonic development to visualize the Ia projection toward the motor pool and to investigate interations between Ia axons and motor dendrites.

Some of our results have been reported in abstract form (Snider et al., 1990, 1991). 

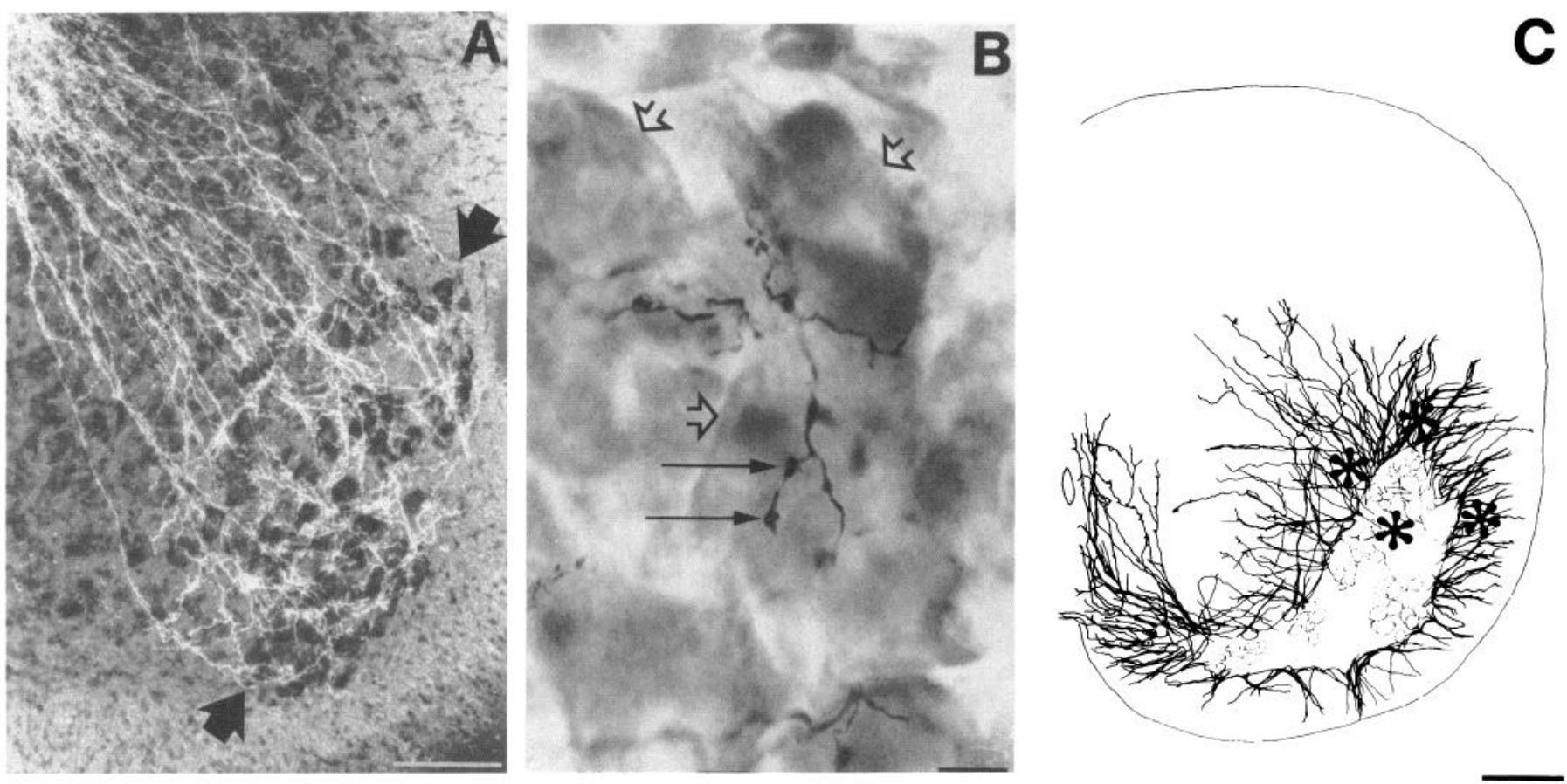

Figure 1. A, Dark-field photomicrograph of DiI-stained fascicles of Ia axons after photoconversion with DAB. This preparation was counterstained with thionin and motor neuron somata appear dark. The dorsal and ventral limits of the motor pools are indicated by the large arrows. Putative boutons were counted in these photoconverted preparations. $B$, High-power view of terminal branches of DiI-stained, photoconverted Ia afferent axons. Swellings larger than $3 \mu \mathrm{m}$ (solid arrows) along these axons were considered to be synaptic boutons. Motor neuron somata are indicated by open arrows. C, Camera lucida tracing of the dendritic projections of motor pools in C5 at PN5. Asterisks show the areas in which bouton counts where made. Note that motor neurons have extensive dendritic projections medially, dorsally, and laterally. Putative boutons were counted in lamina IX in the area of motor neuron somata and proximal dendrites, in lamina VII in the area of the medial dendritic projection, in lamina VII in the area of the dorsal dendritic projection, and in the white matter directly lateral to the motor pool. Scale bars: $A, 100 \mu \mathrm{m} ; B, 10 \mu \mathrm{m} ; C, 200 \mu \mathrm{m}$.

\section{Materials and Methods}

All experiments were done on embryonic (E) and early postnatal (PN) rats. At appropriate stages of pregnancy, females were deeply anesthetized with pentobarbital $(50 \mathrm{mg} / \mathrm{kg})$. Embryos were delivered by cesarean section. At early time points, before E15, embryos were fixed by immersion in fresh 4\% paraformaldehyde. Embryos at E15 and beyond were perfused through the left ventricle with saline followed by fixative. At the later stages, embryos were kept on ice until perfusion in order to maintain the nervous system in good condition.

Animals were postfixed with $4 \%$ paraformaldehyde or formalin for periods ranging from a few hours to several weeks prior to crystal placement. In general, lighter fixation allowed somewhat faster diffusion of the dye. However, even preparations maintained in fixative for weeks or months could be successfully stained.

After the embryos had been postfixed, the cervical spinal cord and dorsal root ganglia (DRGs) with roots intact were removed and pinned in a plastic dish coated with Sylgard (Dow Corning Corp.). The dura was carefully teased away. Motor neuron dendrites and dorsal root afferent axons were stained with either the carbocyanine dye $1,1^{\prime}$-dioctadecyl-3,3,3',3'tetramethylindocarbocyanine perchlorate (DiI; Molecular Probes) or the styryl-pyridinium dye 4-(4-dihexadecylaminostyryl)$N$-methylpyridinium iodide (DiA/4di-16-ASP; Molecular Probes). Crystals of these tracers were repeatedly crushed and a tiny fragment was picked up on the tip of a microelectrode. In order to stain dorsal root axons separately, motor dendrites separately, or both together, several types of preparations were utilized. In many preparations, a crystal was placed in the C5 DRG. At early developmental stages this procedure fully labeled both the spinal projections of DRG cells as well as motor neurons in the $\mathrm{C} 5$ segment. The $\mathrm{C} 5$ motor pools were labeled because of the proximity of the ventral root to the undersurface of the DRG where it joins with the dorsal root to form the spinal nerve. In order to visualize motor pools or dorsal root afferents separately, either the dorsal or ventral root was transected after replacement of the crystal in the DRG. Specimens were kept in fixative at $38^{\circ} \mathrm{C}$ for periods from $3 \mathrm{~d}(\mathrm{E} 15)$ to $17 \mathrm{~d}$ (PN5).
In some cases, DiI and DiA were used in the same preparation. Dil was placed on the dorsal root and DiA on the ventral root. This allowed detailed examination of the relationship between motor neuron dendrites and dorsal root axons, which could be clearly distinguished because they were labeled with different chromophores. In a few preparation, motor neuron dendrites were labeled together with axons in the lateral funiculus. Labeling of funicular axons was accomplished by placing a crystal of DiI in the lateral region of the spinal cord several segments rostral to $\mathrm{C} 5$.

DiI crystals placed in the C5 DRG fully labeled the segmental projection at these early developmental stages. In order to label smaller groups and even single dorsal root axons, two approaches were used. In the first, a particularly small DiI crystal was placed directly on the dorsal root. If the crystal was small enough only a few fascicles of dorsal root axons were stained. In the second method, a crystal was placed in the DRG but most fibers in the dorsal root were transected, interrupting diffusion of the dye. Both of these methods yielded preparations in which only a few of the dorsal root axons in a particular segment were labeled.

After the appropriate time interval, spinal cords were embedded in agar and sectioned on a vibratome in the transverse or parasagittal plane at thicknesses between 70 and $100 \mathrm{~mm}$. Sections were photographed and suitable sections were selected for tracing by camera lucida. The use of black paper and white pencils greatly facilitated the tracing of this fluorescent material. In many sections photoconversion was carried out utilizing 3,3'-diaminobenzidine tetrahydrochloride (DAB; Sandell and Masland, 1988; Bhide and Frost, 1991). For these, sections were incubated for $10 \mathrm{~min}$ at $4^{\circ} \mathrm{C}$ in a solution of $2.5 \mathrm{mg} \mathrm{DAB} / \mathrm{ml} 0.1 \mathrm{M}$ Tris buffer ( $\mathrm{pH} \mathrm{8.2)}$ in the wells of tissue culture plates. The sections were then placed on microscope slides in a small drop of DAB solution. Sections were illuminated using a Nikon Microphot FXA with a $10 \times$ (NA 0.6) objective. The DAB replaced DiI fluorescence with a brown reaction product over periods of $30-90 \mathrm{~min}$ in successful preparations (Fig. $1 A, B$ ).

In order to quantitate the location of terminals of dorsal root afferent axons, direct bouton counts were undertaken in the ventral horn in photoconverted preparations from postnatal animals at ages PN2 and 

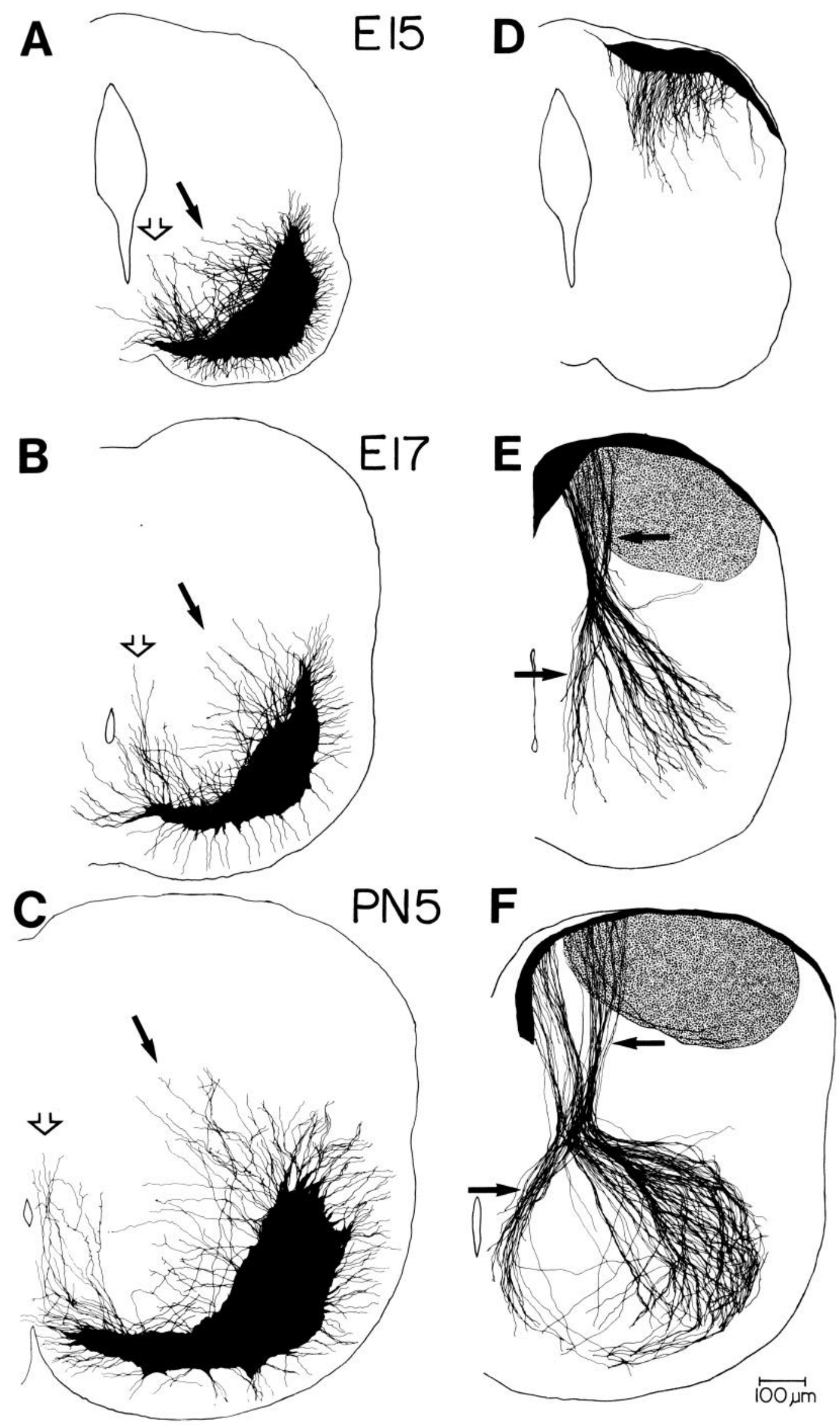
PN5. Some preparations were counterstained with thionin so that the location of motor pools was apparent (Fig. $1 A, B$ ). Other preparations were first viewed at low power. An outline of the motor pool was superimposed on the drawing paper using a representative sample from either PN2 or PN5 in which the motor pool had been stained. A reticule was then placed first within this region (lamina IX) and then immediately adjacent to this region (lamina VII) both medial and dorsal to the motor pool. Finally, the reticule was placed over developing white matter, lateral to the motor pool. The locations of these counting boxes in relation to motor neuron somata and dendrites are shown in Figure $1 C$ (asterisks). The preparations were then viewed under 625 magnification. Any swelling greater than $3 \mu \mathrm{m}$ in diameter along dorsal root axons in the reticule was counted as a bouton (Fig. $1 B$ ). It is realized that this criterion is arbitrary, but it should not introduce any systematic bias affecting synaptic density in the various regions.

Development of motor dendrites, development of dorsal root afferents, and interactions between the two were studied at E13, E15, E17, E19, PN2, and PN5. At least five specimens under each of the conditions at each age were studied. In each preparation, between 4 and 10 sections were drawn, photoconverted, or photographed. Most of the developmental analysis utilized the C4 and C5 segments. However, we studied all major regions of the spinal cord, and some of this material from other regions is shown to illustrate certain points.

Dorsal root afferents in this study were identified solely on the basis of morphology. In mammals, the term "Ia" has traditionally been applied to afferents that innervate the primary endings of muscle spindles in the periphery, project to the ventral horn, and have monosynaptic connections with motor neurons (see Brown, 1981). Although group II fibers from secondary endings of muscle spindles also have monosynaptic contact with motor neurons, intraaxonal fills in cats (Brown, 1981) and our preliminary observations show that these axons traverse the lateral rather than medial portion of the intermediate zone to reach the motor pool. Furthermore, in rat, group II axons appear to be far less numerous than Ia axons (W. D. Snider and L. Zhang, unpublished observations). Thus, the axons described here that project to the graywhite border of the ventral horn and arborize within motor pools can be confidently classified as Ia on the basis of their trajectory and central morphology.

\section{Results}

\section{Development of motor neuron dendrites}

The growth of motor neuron dendrites in the $\mathrm{C} 4$ and $\mathrm{C} 5$ cervical motor pools was studied between E15 and PN5. The C4 segment innervates exclusively axial (paraspinal) musculature, whereas C5 has medially located axial motor pools plus laterally located pools innervating proximal limb musculature.

The development of dendrites in $C 5$ is shown in Figure 2, $A-$ $C$. Different pools of motor neurons are tightly clustered at these early stages, and individual pools were not distinguished in fully stained preparations. By E15, motor limb neurons in the lateral region project dendrites into gray matter (Fig. $2 A$, solid arrow). These dendrites project directly into the path of the incoming dorsal root afferents (Fig. 2D-F). Thus, these dendrites are in a position to influence dorsal root axons coursing toward the motor pools. The pattern of dendritic projection of limb motor neurons changes little between E15 and PN5 (Fig. 2A-C, solid arrows). Dendrites lengthen greatly as the spinal cord grows; however, their relative position in relation to the ventral horn and intermediate zone is maintained.

There are clear differences in the arborizations of motor neurons that subserve axial and those that subserve limb muscles. Medial axial pool dendrites in C5 have dendrites primarily oriented along the gray-white border. As development proceeds, these neurons elaborate dendrites dorsally that course along the midline of the cord (Fig. $2 A-C$, open arrows). Differences in the arrangement of dendrites of motor pools subserving limb and axial musculature are strikingly illustrated by comparing the arrangement in $\mathrm{C} 5$ (Fig. $3 A, B$ ) with the arrangement in $\mathrm{C} 4$ (Fig. $3 C, D)$. Motor neurons in the $\mathrm{C} 4$ spinal segment do not innervate the limb and therefore are exclusively devoted to axial musculature. The axial motor neurons do not project dendrites into gray matter and maintain the orientation of their dendrites primarily along the gray-white border at least as late as PN5 (Fig. $3 C, D$, solid arrows). There is a striking tendency for these dendrites to fasciculate into prominent bundles (Fig. $3 C, D$, solid arrows).

At early developmental stages, there is a prominent projection of dendrites from both groups of motor neurons into the developing lateral and ventral funiculi. 'This projection is not the result of random dendritic outgrowth. We frequently observed dendrites making sharp turns to gain access to the funiculi (Fig. $3 E$ ), whereas dendrites that project into gray matter are straight. This funicular projection becomes increasingly dense between E1 3 and E19 and is still present as late as 1 week postnatally, although increasing numbers of funicular axons separate the dendrites from the lateral margin of the cord. The proximity of white matter dendrites to funicular axons is shown in a longitudinal section of cervical spinal cord at E17 where both motor neurons and axons in the lateral funiculus were stained (Fig. $3 F$ ). The motor pool is indicated by long solid arrows, motor dendrites by short solid arrows, and funicular axons by open arrows. It is appealing to hypothesize that these white matter dendrites have a special relationship to funicular axons. In support of this idea is that they receive no innervation from dorsal root afferents (see below).

\section{Development of the dorsal root projection to motor pools}

Figure 2, $D-F$, shows the development of the dorsal root projection to motor pools in the C5 segment. Dorsal root afferent axons first penetrate gray matter at E15 (Fig. $2 \mathrm{D}$ ). Observations in the parasagittal plane show that afferents destined for motor pools arise as "interstitial nodal branches" (see O'Leary and Terashima, 1988) from dorsal root axons running rostrocaudally in the developing dorsal columns. Axons enter gray matter in fascicles of 4-10 axons each. Between E15 and E17 these fascicles converge in the intermediate zone in the area of Clark's column and fan out again en route to the motor pools (Fig. 2E).

\footnotetext{
Figure 2. $A-C$, Development of motor neuron dendrites in C5. $A, \mathrm{E} 15$. The dendritic projection of limb motor neurons in gray matter (solid arrow) is already well established at this age. There is also a dense projection into developing ventral and lateral funiculi. $B$, E17. Dendrites of lateral motor neurons (solid arrow) project directly in the path of incoming Ia afferents. Dendrites of medial motor neurons project dorsally along the midline (open arrows in $A$ and $B$ ). $C$, PN5. Despite cord growth, the overall pattern of dendritic outgrowth of limb (solid arrow) and axial (open arrow) motor neurons has changed little since E15. D-F, Development of the Ia projection in C5. $D$, E15. Dorsal root afferents first penetrate gray matter. E, E17. Muscle afferents grow in fascicles toward the motor pool. Most are unbranched at this age. They grow to but do not cross the graywhite border. In general, spatial relationships apparent on exit from the intermediate zone are maintained en route to the motor pools. Afferents destined for medially located axial motor pools (arrows) enter the cord laterally and cross over the other afferents in the intermediate zone to grow along the midline to their targets. $F$, PN5. The overall arrangement is similar to that at E17. Tertiary and higher-order branching is well established by this age. Note the medial ventral horn that contains commissural neurons receives few dorsal root afferents. Dense labeling of the dorsal horn in these preparations is indicated by the stippling.
} 

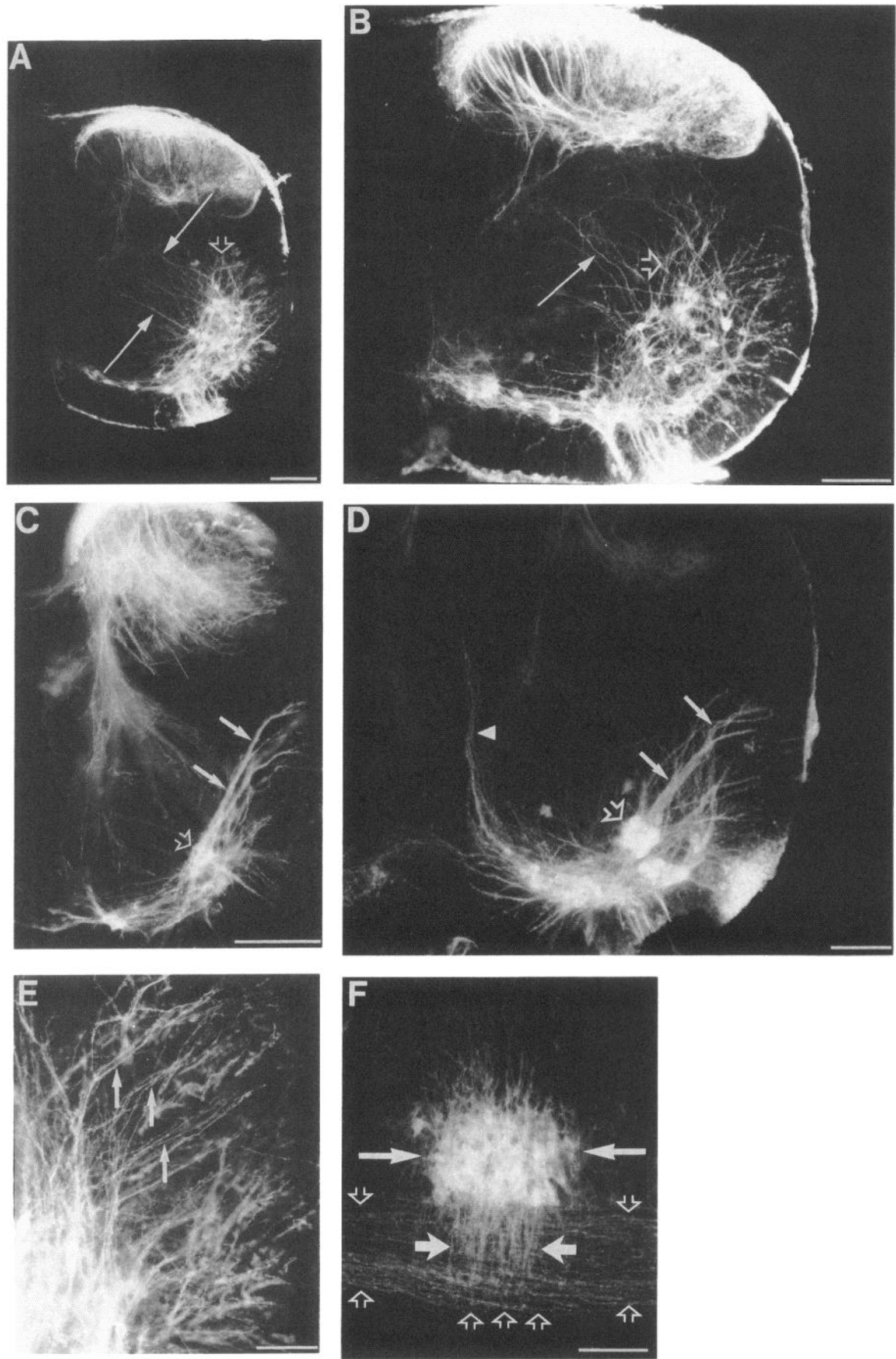
This convergence of fascicles in the arca of Clark's column is striking (Fig. $4 B$ ) and suggests that Ia axons are either attracted to this region or inhibited from traversing more lateral regions of the intermediate zone.

Projection of these fascicles toward the ventral horn is not a random process. A rough topography is present between fascicles destined for medially located axial motor pools and laterally located pools projecting to limb muscles. Thus dorsal root afferents destined for these two spatially separate groups take entirely different pathways. Axons destined for the medially located axial motor pools segregate from others in the intermediate zone and grow along the midline toward their target neurons (Fig. $2 E, F$, arrows).

It is possible that the region of ventral horn to which medial versus lateral fascicles project is unrelated to the group of cells they ultimately innervate. In order to examine the relationship between trajectory of a fascicle in the intermediate zone and location of its terminal arbor in the ventral horn, we prepared specimens in older animals in which only a few of the fascicles in the segmental dorsal root projection were labeled (see Materials and Methods). The significance of these different trajectories was apparent in preparations where individual axon arbors could be resolved (Fig. $5 A$ ). The medially projecting axons (open arrows) clearly innervate an entirely different group of target cells than afferents that take a lateral trajectory (solid arrows).

The projection of Ia afferents to the laterally located limb pools also exhibited some degree of spatial order. Examples of the spatial order of this projection are shown in Figure 4 at low power $(A-C)$ and at higher power $(D-G)$. The fascicles emerge from an area in the medial intermediate zone that receives dense innervation from dorsal root afferents and presumably represents Clark's column or an analogous group of spinoccrebcllar cells (Fig. 4B, long solid arrow). The most dorsal and most ventral fascicles at different ages are delineated by the large arrows in Figure 4, D-G. A spatial relationship among the bundles is apparent as they leave the intermediate zone and is roughly maintained en route to the motor pools. The order among fascicles is not perfect as axons or entire fascicles sometimes cross one another en route to motor neurons. However, in general, more dorsal bundles project to dorsally located motor pools and more ventral bundles project to more ventrally located motor pools (Fig. $4 D-G$ ).

In order to assess further the significance of this apparent order in the projection of fascicles to the lateral motor pools, we studied the $\mathrm{C} 8$ cervical segment in which motor pools are widely separated along the dorsal-ventral axis because of the prominent projection to the forelimb (Fig. $5 B-E$ ). These prep- arations were photoconverted and counterstained with thionin in order to visualize motor neurons. Motor neuron somata are indicated. Two ages, E17 ( $B$ and $C$ ) and PN2 ( $D$ and $E$ ) where studied. In examining the Ia projection at E17, it is apparent that fascicles project to different regions of ventral horn from the outset (Fig. 5B,C). These different initial trajectories appear to have significance for connectivity in older animals. Although there is considerable intermingling at PN2, it is clear that more ventrally projecting fascicles innervate a different set of targets than more dorsally projecting fascicles (Fig. $5 D, E$ ). Thus, it does not appear that fascicles initially project to one region and then leave that region to innervate widely separated motor neurons located elsewhere. It is important to emphasize that the identity of axons that make up these fascicles is unknown. Assessing the extent to which the Ia projection to the lateral pools is topographic will require assessing the pathways of identified la axons from different muscles.

It is possible that the position of fascicles en route to the ventral horn is related to the position in which they leave the dorsal columns. This idea was difficult to assess in our material because of dense staining of the dorsal horn. However, it was apparent that afferents to medial axial motor pools enter gray matter most laterally and cross over other afferents in the intermediate zone (Fig. 2E,F, arrows). Thus, in six preparations in which the dorsal horn was lightly stained, the major bundle along the midline could be seen to arise from fascicles leaving the dorsal columns in the lateral $25 \%$ of the Ia axon entry zone (see also Réthelyi and Szentágothai, 1973).

Ia axons are clearly inhibited from growing in certain regions of the developing spinal cord. The most striking example of this is that Ia axons virtually never enter developing white matter despite their proximity to lateral regions of the cord. By E17, some fascicles of axons have reached the gray-white border (Fig. $2 E$ ). There is a sharp demarcation between the axons of the dorsal root projection and the developing funiculi (Fig. 4A$C, H)$. The exclusion of Ia axons from developing white matter is strikingly illustrated in the parasagittal orientation (Fig. $4 H$ ). When bundles of axons reach the gray-white border, they defasciculate and axons turn and grow within the motor pools rather than along dendrites coursing in the developing funiculi.

Fascicles of dorsal root axons also appear to be inhibited from growing in the medial part of the ventral horn (Fig. $4 A-C$ ). This region contains many spinal interneurons with axons in the ventral commissure (Fig. 4C, solid arrow; also I. Silos-Santiago and W. D. Snider, unpublished observations). That dorsal root axons are actively excluded from this region is suggested by the trajectory of afferents to axial motor pools. Rather than growing straight through the intermediate zone and ventral horn to their

Figure 3. Differences in dendritic arborization between axial and limb motor pools. $A$ and $B$, Motor pools in C5 at PN5. These motor neurons project long dendrites (arrows) in gray matter directly in the path of incoming dorsal root afferents. These neurons also have prominent dorsally projecting dendrites (open arrows), some of which reach almost to the dorsal horn. $C$, Motor pools in $C 4$ at E17. Strikingly oriented and fasciculated dendrites (solid arrows) from a clustered group of motor neurons (open arrow) can be seen. These dendrites curve toward the white matter in the lateral region of the cord. $D$, Motor pools in C4 at PN5. The overall arrangement of dendrites is similar to that at E17. Dendritic bundles (solid arrows) emerging from a cluster of motor neurons (open arrow) are shown. Few dendrites project into gray matter in the path of incoming dorsal root afferents. Note that dendrites of the most medial neurons grow dorsally along the midline (arrowhead). $E$, Higher-power view of fasciculated dendrites in white matter. The white matter dendritic fascicles are composed of three or four individual dendritic branches (arrows). Note the sharp turns some of these dendrites make toward the funiculi. The edge of the cord is at the edge of the photograph. $F$, Motor dendrites projecting in the path of lateral funicular afferent axons. Both motor neurons (long solid arrows) and funicular afferents were labeled. The spinal cord was sectioned longitudinally. The motor dendrites (short solid arrows) in developing white matter project directly in the path of descending funicular axons. Funicular axons course through the area delineated by the open arrows. Scale bars: $A-D, 200 \mu \mathrm{m} ; E, 25 \mu \mathrm{m} ; F, 100 \mu \mathrm{m}$. 

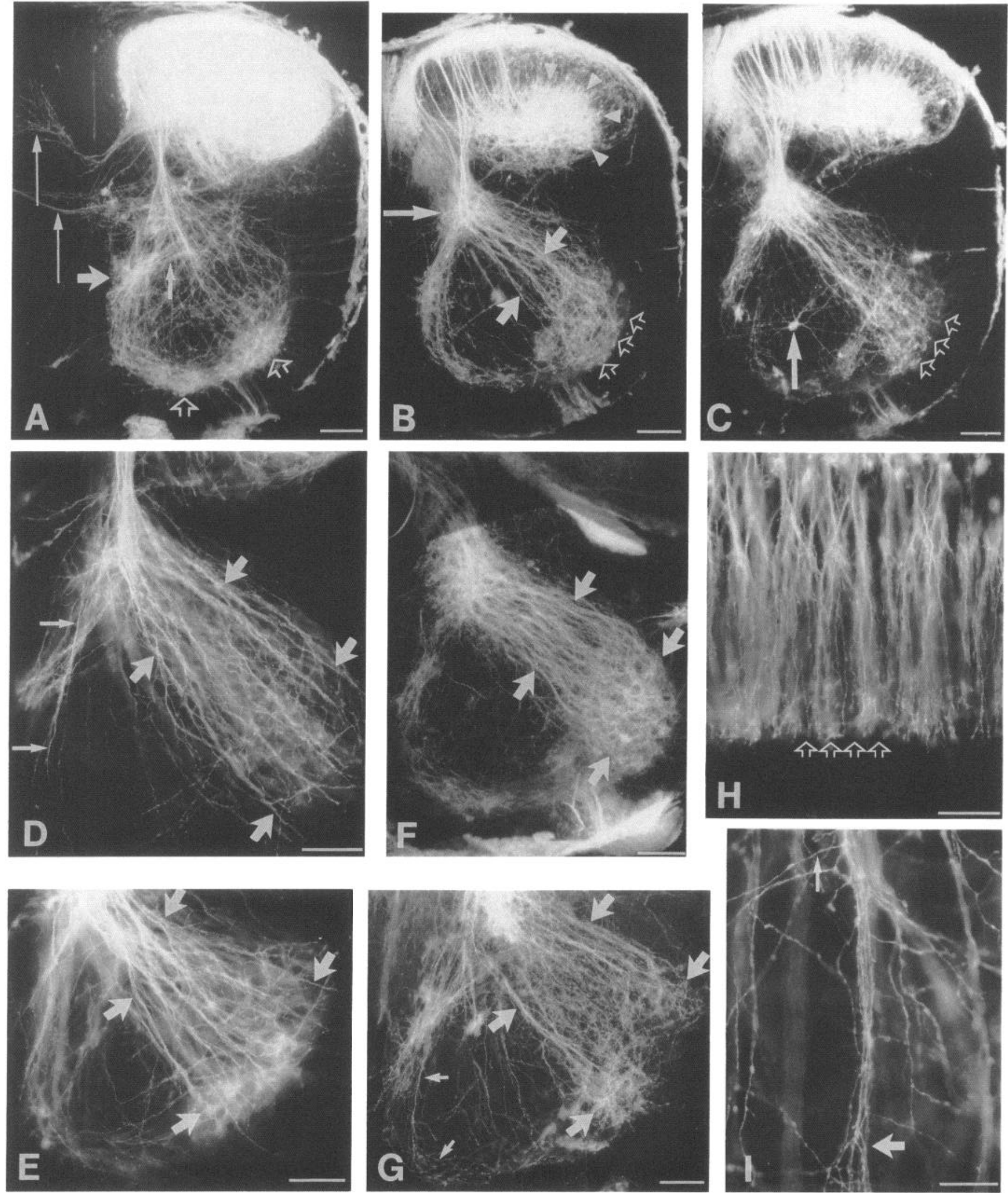

Figure 4. Fasciculation and spatial order of dorsal root afferent axons. $A-C$, Low-power views of afferent axon fascicles projecting to the motor pools in $\mathrm{C} 4$ and $\mathrm{C} 5$ at PN2. A, C4. Fascicles project to the motor pools (open arrows) with a clear spatial order. Some axons make a sharp turn in the intermediate zone (small solid arrow) to a cell group along the midline (large solid arrow). Two groups of axons cross the midline in this region (thin vertical arrows). B, C5. Several well-delineated fascicles between the short, solid arrows are apparent. Afferents stop at the gray-white border (open arrows). There is dense branching in the area of a dorsal midline cell group (long solid arrow). The distribution in the dorsal horn of afferents from hair follicles and other low-threshold mechanoreceptors is delineated by arrowheads. $\mathrm{C}, \mathrm{C} 5$. A central area in intermediate zone and ventral 
target motor neurons, these axons grow along the midline and then curve laterally to innervate axial motor neuron cell bodies (Fig. 5A, open arrows).

Most dorsal root axons projecting to the ventral horn remained fasciculated until E17. Starting at E17, branches are elaborated by individual axons with increasing intensity and defasciculation occurs close to the motor pool. By E19 many axons exhibit extensive tertiary and higher-order branching as well as bouton formation within the motor pools (see below). The location of these axons in the ventral horn as well as the proximity of their terminal arbors to motor neurons allow us to classify them as group Ia (see also Brown, 1981; Kudo and Yamada, 1987; Ziskind-Conhaim, 1990).

We have incidentally observed the development of other types of dorsal root afferent fibers. Some axons with a morphology compatible with group II axons (data not shown) have a more lateral trajectory to the ventral horn. These are less numerous than la axons. Other groups of afferents also have well-developed projections by E17 (see also Smith, 1983). Some of these enter medially in fascicles, project to laminae III and IV, and presumably include afferents from hair follicles and other cutaneous and subcutaneous mechanoreceptors (Fig. 4B, arrowheads). Fine cutaneous afferents to the superficial dorsal horn first enter the cord in the cervical region at E17. Substantial ingrowth of axons in the superficial dorsal horn is apparent by by F.19. By PN5, the superficial dorsal horn is densely innervated (see also Fitzgerald, 1987; Ruit et al., 1992). In the upper cervical region, two classes of afferents cross the midline (Fig. $4 A$, thin vertical arrows).

There are at least two targets for dorsal root afferent axons along the midline in the $\mathrm{C} 4$ and $\mathrm{C} 5$ segments (Figs. $4 A, B ; 5 A$ ). The most dorsal of these is presumably a group of spinocerebellar cells analogous to Clark's column (Figs. $4 B$, long solid arrow; $5 A$, top arrowhead). Branching toward this target is shortrange and begins on E17. Many of these branches arise from the Ia muscle afferents. This dorsal target is located precisely in the area where afferents fan out on their way to the motor pool. There is also dense bouton formation more ventrally on a group of cells that has not yet been identified (Fig. $4 A$, large solid arrow; Fig. $5 A$, bottom arrowhead). Dorsal root axons reach this ventral midline group between E16 and E17. Some afferents reach this target after making a sharp turn in the intermediate zone at E1 7 and appear to have this midline group as a primary target; others arise as $90^{\circ}$ branches from Ia afferents en route to the motor pools (Figs. $4 A$, small solid arrow; $5 A$ long arrow).

\section{Interactions between dorsal root afferents and motor neuron dendrites}

Utilizing preparations where dorsal root afferents were stained with DiI and motor neurons stained with DiA, we studied interactions between dorsal root axons and motor dendrites in these embryonic and early postnatal animals. In particular, we asked whether motor neuron dendrites influence the trajectory of ingrowing Ia afferents en route to particular regions of the developing ventral horn.

Examples of these double-label preparations traced with camera lucida are shown in Figure 6. We have compared a segment subserving axial musculature, $\mathrm{C} 4$, with a segment innervating limb musculature, L5. These observations were clear-cut in C4. These neurons have most of their dendrites oriented along the gray-white border (see above) and project few dendrites directly into gray matter. Examples of afferent-dendrite interactions in $\mathrm{C} 4$ at $\mathrm{E} 16$ and $\mathrm{E} 19$ are shown in Figure $6, A$ and $B$. In C4, afferent fascicles leaving the intermediate zone project more or less straight to different regions of the ventral horn. The spatial order of the projection is apparent. Contact with motor neuron dendrites is clearly not required in order for these afferents to reach the vicinity of motor neuron cell bodies. In fact, these dorsal root afferents traverse the entire dorsoventral length of the cord prior to contacting the somata and dendrites of motor neurons in the most ventral region.

The pattern of afferents projecting to limb motor neurons is more complex. The segment of cord with the densest projection of dendrites in gray matter is L5, and we have therefore illustrated afferent-dendrite interactions in that segment (Fig. 6C,D). The first interactions between these dorsal root afferents and motor neuron dendrites can be observed at E17. Motor neurons in the limb region have extended long dendrites in gray matter by this age, and these dendrites are deployed in the path of the incoming dorsal root afferent fascicles (Fig. 6C). Although there are instances where afferent growth cones appear to contact dendrites and occasional instances where afferents grow along dendrites for a significant distance, many axon fascicles do not parallel motor dendrites. This is particularly apparent for axons projecting to more ventrally located motor pools. Their dendrites are nearly parallel to the mediolateral axis, whereas afferents project at an angle of $45^{\circ}$.

By E19 there is an intense area of overlap between dorsal afferents coursing toward the motor pools and motor neuron dendrites projecting dorsomedially in the intermediate zone (Fig. $6 D$ ). Nevertheless, double-label preparations revealed long stretches of dendrites not contacted by afferents. The major impression one gets from the anatomical material is that growing afferents pass over distal dendrites en route to proximal dendrites and somata.

The idea that these gray matter dendrites do not influence the trajectory of Ia fascicles is supported by the location of their terminal branches and boutons (Fig. 7). Most Ia axons do not defasciculate and elaborate boutons until they are near motor neuron somata at $\mathrm{E} 17$ (Fig. $7 \mathrm{~A}$ ). Between $\mathrm{E} 17$ and $\mathrm{E} 19$ there is dense branching and bouton formation in the area of the motor pools (Fig. 7B). In these double-label preparations it was clear that many of the branches and boutons are in the region of somata and proximal dendrites, even in the postnatal period

horn receives little innervation from dorsal root afferents at this stage. A neuron in this region with an axon in the ventral commissure is shown (solid arrow). $D-G$, Higher-power view of fascicles projecting toward the motor pool at different ages: $D$, E17; $E$, E19; $F$, PN2; $G$, PN7. A rough spatial order is apparent as fascicles leave the intermediate zone. Dorsal fascicles (upper large arrows) clearly project toward the dorsally located motor pools. Ventrally located fascicles (lower large arrows) project to more ventrally located pools. Small arrows in $D$ and $G$ denote afferents projecting to medially located axial pools. $H$, Fascicles of Ia afferents from $\mathrm{C} 5$ in the parasagittal orientation at E19. The region shown is between Clark's column in the interemdiate zone and the ventral edge of the cord. Note the sharp demarcation between fascicles of la afferents and developing white matter (open arrows). I. High-power photomicrograph of a fascicle of Ia afferents in the transverse plane. Dorsal is toward the top. A branch some distance from the motor pool (small arrow) is given off from a node along the parent axon. Axons are defasciculating near the motor pool (large arrow). Scale bars: $A-C, 125 \mu \mathrm{m} ; D-H, 100 \mu \mathrm{m} ; I, 25 \mu \mathrm{m}$. 

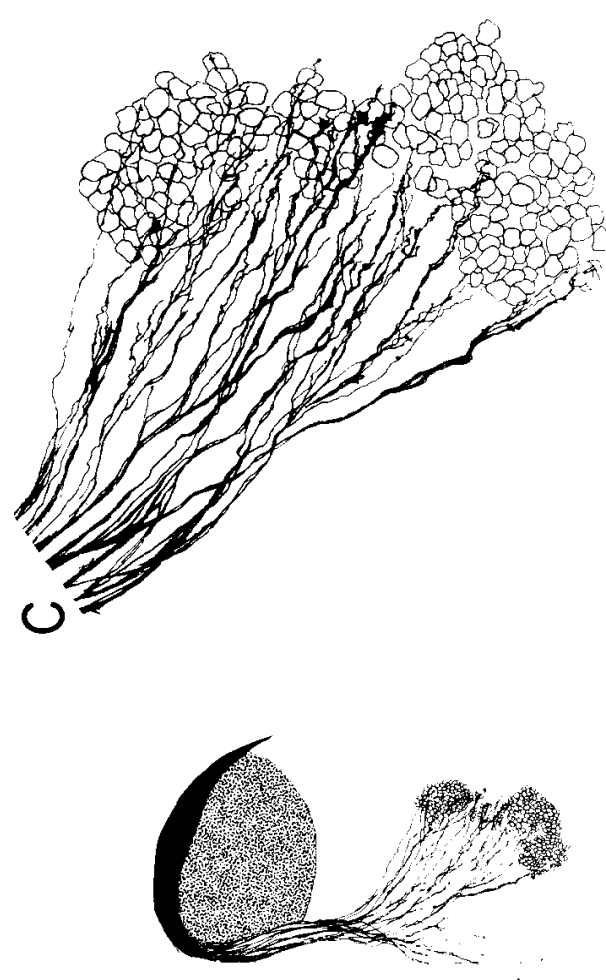

$\infty$

山

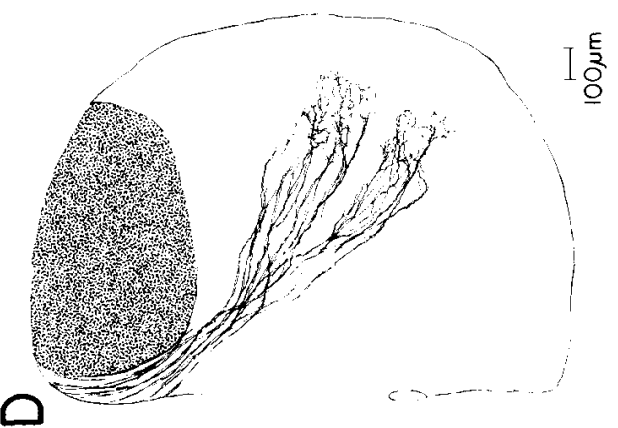

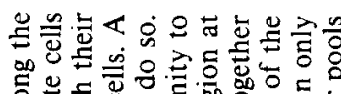

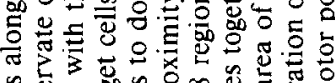

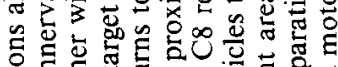

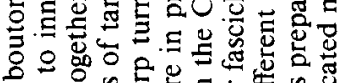

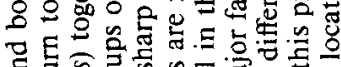

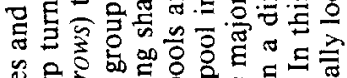

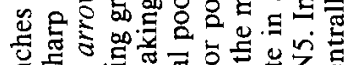

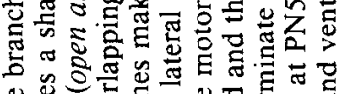
곤

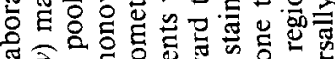

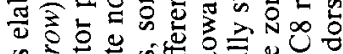

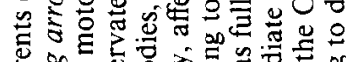

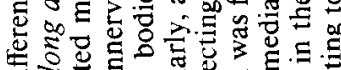
ब

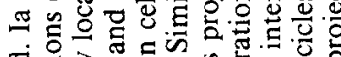

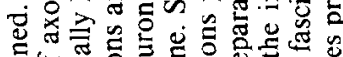

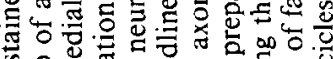

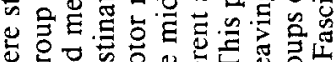

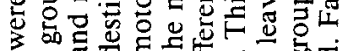

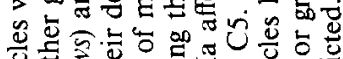

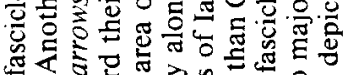

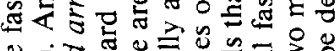

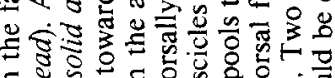
콩

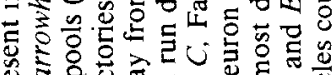

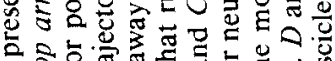

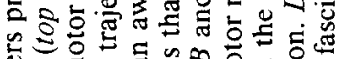

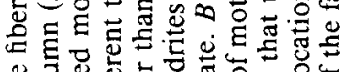

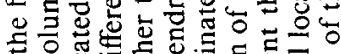

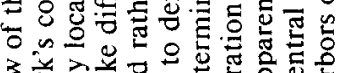

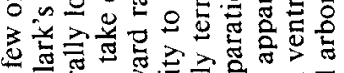
๙ 궁워

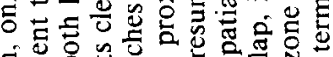

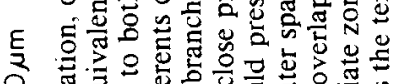

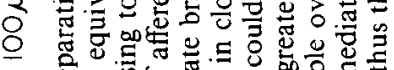

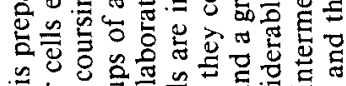

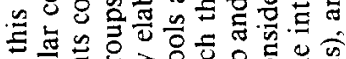

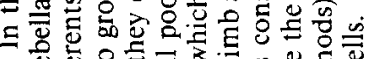

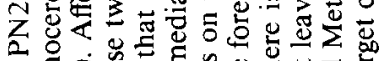

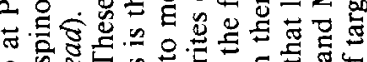
की

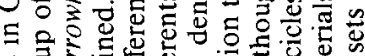

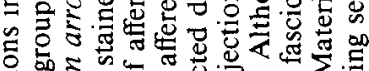

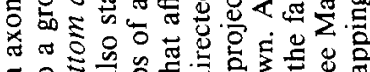

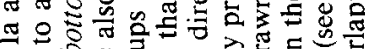

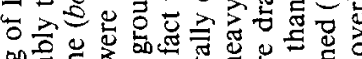

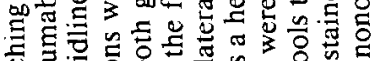

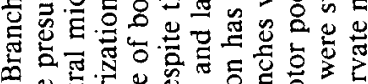
ه.气

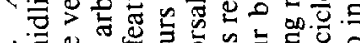

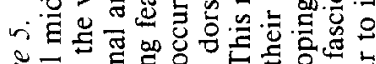

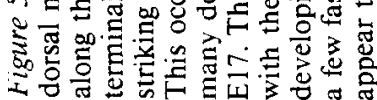


(Fig. $7 C, D$ ). Thus, despite the fact that Ia afferents course in the intermediate zone in an area richly populated with motor dendrites (lamina VII), much of the terminal branching and bouton formation occurs in lamina IX near motor neuron somata. Direct bouton counts from photoconverted preparations in the $\mathrm{C} 5$ segment showed that lamina IX contained twice the number of boutons per unit of area as an area of lamina VII directly medial to the motor pool that contains many motor dendrites (Table 1). Few boutons were found within lamina VII dorsal to the motor pool despite the fact that many motor dendrites project in this region (Figs. $1 C$; Fig. $3 A, B$, open arrows; $7 B, D$, upper right arrows). Virtually no boutons are found on dendrites that project in white matter.

Additional evidence for the importance of proximal innervation is shown by examining the behavior of individual Ia fascicles. These could be visualized in preparations where particularly small crystals of DiI were utilized, or where many dorsal root axons were interrupted proximal to the area of crystal placement (see Materials and Methods). Figure $8, A-C$, shows the behavior of individual fascicles of Ia axons (solid arrows). Defasciculation and elaboration of boutons are shown near the gray-white border (open arrows), obviously in proximity to motor somata. Figure $8, D$ and $E$, shows examples of individual Ia axons (thin arrows). Terminal arbors (short arrows) arc claborated in the most ventral region of the cord near the graywhite border, and thus must be in proximity to motor neuron somata. Note that there is little branching in lamina VII. Figure $8, F$ and $G$, shows two examples of individual axon arbors in preparations where motor neurons were also stained. The proximal location of the innervation is apparent. In Figure $8 G$, note that Ia axons do not grow out along the dendrite fascicles (right open arrows) that project dorso-laterally.

Finally, evidence for the importance of proximal innervation is shown by the behavior of axons that initially project dorsal or medial to the motor pools. These axons invariably turn and elaborate branches toward motor neuron somata. For example, bundles that project dorsal to the lateral motor pools either elaborate branches or turn directly ventrally toward the cell somata, sometimes at an angle of almost $90^{\circ}$ (Fig. $5 A$, solid arrows). This occurs despite the fact that many motor ncurons
Table 1. Counts of putative boutons in different regions in the $C_{5}$ segment

\begin{tabular}{llc} 
Location & $n$ & $\begin{array}{c}\text { Varicosities/ } \\
1000 \mu \mathrm{m}^{2}\end{array}$ \\
\hline Lamina IX & 7 & $11.3 \pm 1.3$ \\
Lamina VII (medial) & 7 & $5.9 \pm 0.7^{*}$ \\
Lamina VII (dorsal) & 7 & $2.2 \pm 0.9^{*}$ \\
White matter & 7 & $0.1 \pm 0.1^{*}$
\end{tabular}

$\overline{\text { Swellings greater than } 3 \mu \mathrm{m} \text { in diameter along stained dorsal root axons were }}$ counted in four regions that contain dense projections of motor neuron dendrites: (1) lamina IX, which is the area of motor neuron somata and proximal dendrites; (2) lamina VII directly medial to lamina IX; (3) lamina VII dorsal to the motor pool; and (4) white matter directly lateral to the motor pool. The values given are the means $\pm \mathrm{SD}$.

* Significant difference from value in lamina IX $(p<0.005$ by Student's $t$-test).

have prominent dorsally directed dendrites that could receive this innervation (Figs. $1 C ; 3 A, B ; 7 B, D$ ). Similarly, axons running along the midline destined for axial motor pools grow and branch almost directly laterally toward axial motor neuron somata (Figs. $5 A$, open arrows; $7 B$, horizontal arrow). None of these axons grow dorsally along the extensive dendrites of axial motor neurons that project along the midline (Figs. $2 \mathrm{C}$, open arrow; $3 D$, arrowhead).

\section{Discussion}

\section{Early outgrowth of motor neuron dendrites}

In this work, we have traced the growth of dendrites of cervical motor neuron pools from E15 through the first postnatal week. There are interesting differences in the pattern of arborization between neurons that subserve axial musculature and neurons that subserve limb muscles. In general, the axial motor neurons occupy a medial or ventral position in the motor pools. The dendrites of these neurons tend to extend initially along the developing gray-white boarder and form tightly fasciculated bundles. Rostrocaudal dendritic bundling has been previously described as a prominent feature of some motor pools (Scheibel and Scheibel, 1966b). The precise function of this dendritic fasciculation is not known, but it must provide for easy acti-

\begin{abstract}
Figure 6. Interactions between afferent fascicles and motor dendrites in double-label preparations. These are camera lucida tracings from fluorescent material where DiI-stained dorsal root afferents are rendered in black and DiA-stained motor neurons are rendered in red. An area with few dendrites in gray matter $(\mathrm{C} 4, A$ and $B$ ) is compared with an area with dense dendritic projections into gray matter (L5, $C$ and $D$ ). $A, \mathrm{C} 4$ at E16. Few motor dendrites project into gray matter in this segment. A spatial order is apparent even at this early age, and fascicles of afferents are projecting toward appropriate regions of the ventral horn. B, C4 at E19. Note a rough topography with the laterally, ventrally, and medially projecting fascicles arriving in different locations in the ventral horn without guidance from motor neuron dendrites. $C$, L5 at E 17 . Fascicles of Ia afferents project toward the motor pool. Note that afferents appear to pass over distal motor dendrites toward the main mass of neurons. $D$, L5 at E19. Although there is a dense area of overlap, afferents do not stop when entering the region where dendrites project, but rather pass through this region to the area of motor neuron cell bodies. Furthermore, afferents do not appear to course along dendrites on their path to the motor pool. Conversely, long stretches of dendrites are clearly not associated with these dorsal root afferents. Labeling in the dorsal horn was dense in these preparations and is indicated by the stippling.
\end{abstract}

Figure 7. Interaction between Ia afferents and motor neuron dendrites in C5. Afferents were stained with DiI, and motor neurons with DiA. A, Relation of Ia fascicles to motor neuron somata at E17. Fascicles of Ia axons (long arrows) project directly to clusters of motor neuron somata (open arrows). These axons are unbranched at E17. B. Ia afferents and motor neurons at E19. The yellow area within the motor pool is the area of densest terminal branching and bouton formation. Note that motor neuron dendrites projecting dorsally and laterally are not innervated (short vertical arrows) and no dorsal root axons penetrate the white matter. There is a large central area that is not traversed by these axons (large arrow). Afferents to axial pools avoid this region by traveling along the midline, then curving laterally (horizontal arrow) to innervate motor neurons. The dense area of staining along the midline represents inadvertent labeling of radial glia. $C$. Higher-power view of a double-label preparation at PN2. Not all fascicles were stained. Note that fascicles in the focal plane (solid arrows) are predominantly unbranched prior to reaching the motor pool. Terminal branching (orange) is in the area of the motor neuron somata. Open arrows indicate the border between motor neurons and the developing funiculi. $D$, Fully stained preparation at PN2. There is heavy branching and bouton formation in the area of the motor pool (orange). No Ia afferents project in white matter (open arrows) or to dorsally directed dendrites (solid arrows). Scale bars, $100 \mu \mathrm{m}$. 

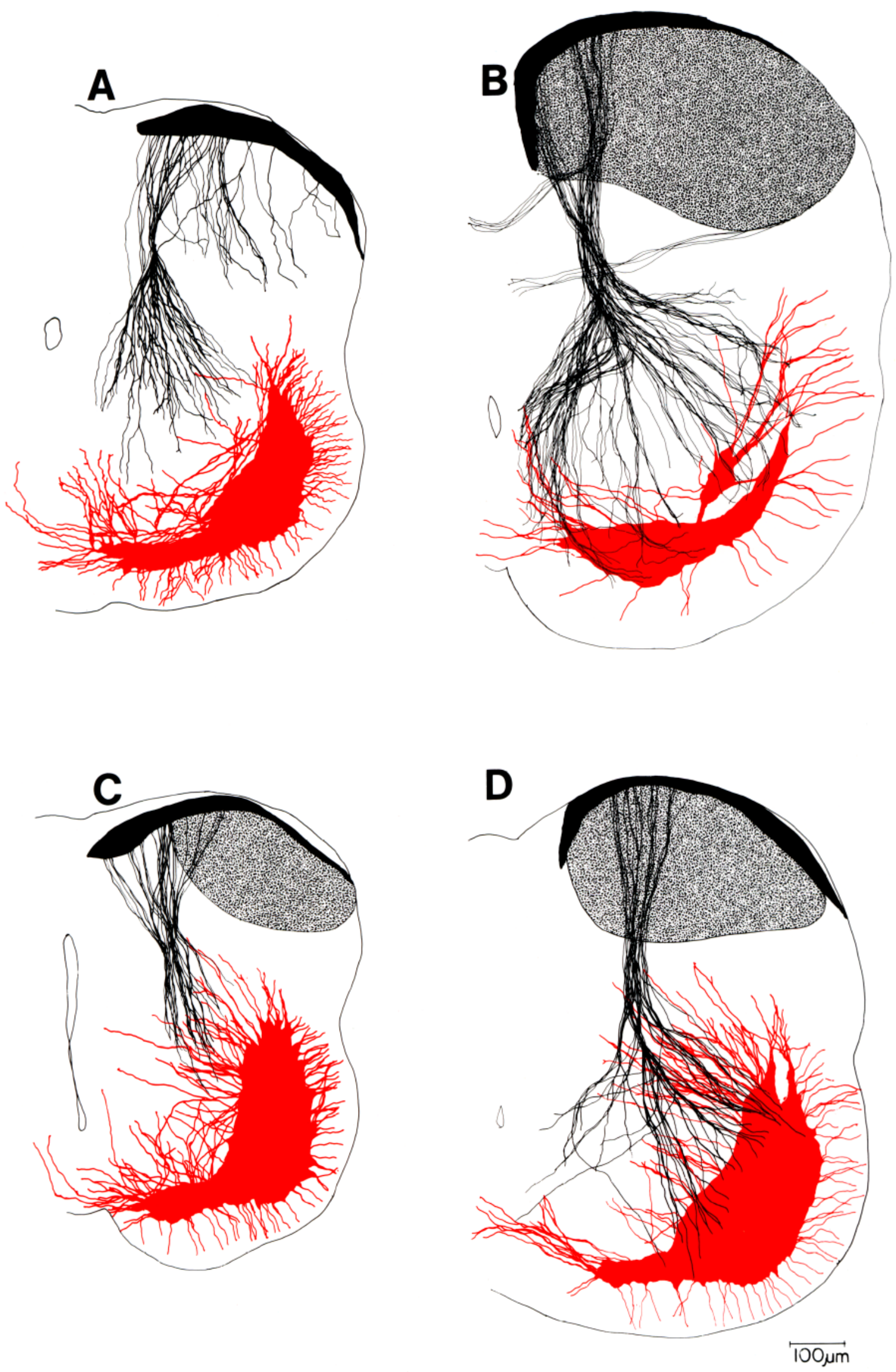


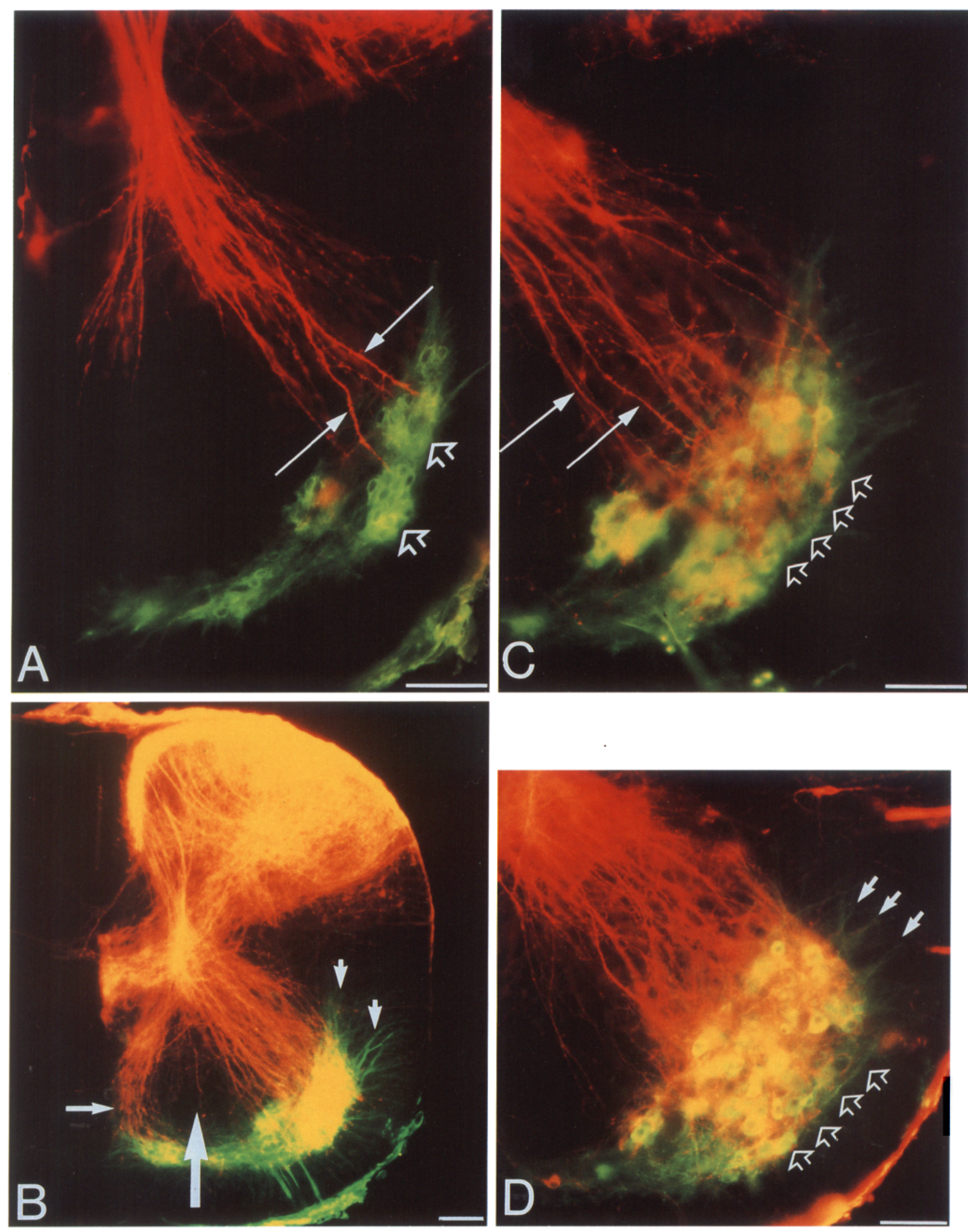



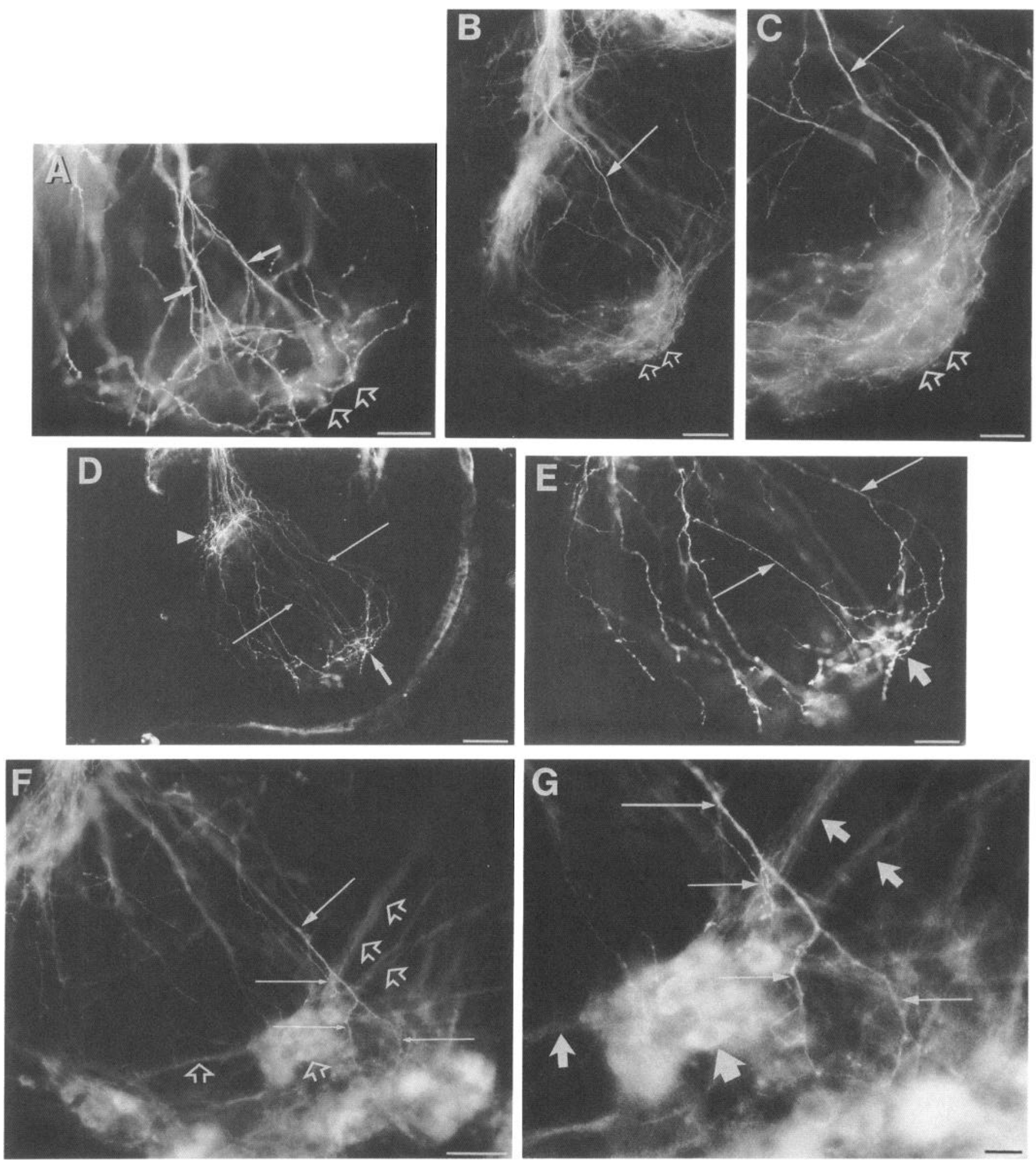

Figure 8. Single fascicles and individual Ia axons arborizing near the gray-white border. $A$, Defasciculation occurring near motor neuron somata at E19. Two fascicles separating into individual axons are denoted by solid arrows. Terminal branches and boutons are in the region of the graywhite border (open arrows) obviously in proximity to motor neuron somata. $B$ and $C$, Low- and high-power photomicrographs of a single fascicle (thin arrows) at E19 shown from its origin in the intermediate zone. This fascicle elaborated terminal branches and boutons in proximity to motor neuron somata, which are faintly outlined. The gray-white border is again indicated by open arrows. Note that no branches are elaborated in medial ventral horn. $D$, Single axons projecting to the motor pool at PN2. Note the branches in the intermediate zone (arrowhead) presumably to spinocerebellar cells (Clark's column). Long individual axons (thin arrows) terminate in arbors near the gray-white border (short arrow). Terminal arbors do not penetrate developing white matter. $E$, Axons in $D$ are shown at higher power. $F$ and $G$, An individual fascicle and its relationships with motor dendrites shown at low $(F)$ and high power $(G)$ in C4 at PN5. Several areas of putative contact between afferent axons (thin arrows) and a pool of motor neurons are shown. Major dendritic bundles are indicated by open arrows in $F$ and solid arrows in $G$. Note that putative contacts are on very proximal regions of the dendrites near the cell somata. Scale bars: $A, C$, and $E, 50 \mu \mathrm{m} ; B, D$, and $F, 100 \mu \mathrm{m} ; G, 25 \mu \mathrm{m}$. 
vation of an entire motor pool, as opposed to individual neurons. Axial motor pools do not project dendrites in gray matter in the path of incoming dorsal root afferents.

The dendrites of neurons projecting to the limb region have a different pattern. A prominent and early feature of their dendritic arborization is a projection into gray matter either medially or dorsomedially directly in the path of incoming dorsal root afferents. Interestingly, in the cervical region this gray matter projection is well established by E15, the first day in which dorsal root afferent axons can be seen entering the spinal cord. This is in contrast to the developmental sequence reported in amphibia (Jackson and Frank, 1987), and in some previous studies in mammals (Vaughn et al., 1988).

Another prominent feature of early dendritic growth in both limb and axial regions is the tendency for dendrites to grow into the developing white matter. Between these fasciculated dendrites run axons of the various afferent systems in the funiculi. It is an attractive hypothesis, though it remains to be proven, that funicular axons utilize contact with white matter dendrites to gain entrance into the gray matter of the spinal cord at the appropriate segment. Whatever the function of these white matter dendrites, they are not contacted by dorsal root afferents that appear to be actively excluded from developing funiculi. The mechanism for this exclusion was not investigated, but it seems likely that either radial glial foot processes or descending afferent axons express molecules that inhibit dorsal root afferent axon growth. This exclusion of dorsal root afferents from the developing funiculi has the implication that funicular axons have exclusive access to the motor neuron dendrites that project in this region.

\section{Ia afferents project toward the ventral horn in spatially ordered fascicles}

A feature of the Ia projection in mammals that has not been previously appreciated is that Ia axons project toward the ventral horn in spatially ordered fascicles. It has not previously been recognized in the Ia-motor neuron system presumably because its demonstration required the ability to stain the Ia projection fully. A rough topography is present at least between laterally and medially located motor pools. Bundles destined for medially located axial pools separate from other fascicles high in the intermediate zone and grow along the midline to the axial motor pool. They thus take an entirely separate pathway to their target neurons than fibers destined for limb motor neurons. Our data are consistent with the idea that a topography may also be present in the projection to the lateral pools, but this issue will require further study.

That mechanisms exist to promote an orderly projection of Ia axons to the ventral horn may not be surprising in view of the sterotypical location of motor pools in mammals (Romanes, 1964; McHanwell and Biscoe, 1981; see also Tyrrell et al., 1990) and in view of the somatotopy exhibited by other classes of dorsal root afferents (reviewed in Willis and Coggeshall, 1991; see also Hongo et al., 1987). Thus, the Ia projection may bear more similarity than previously supposed to topographically ordered projections in other parts of the mammalian nervous system (Udin and Fawcett, 1988). An implication of our interpretation is that axons within individual fascicles should be from the same or closely related muscles in the periphery. It should be possible in the future to test this hypothesis by careful labeling from individual muscles rather than the dorsal root.

\section{Interactions between Ia axons and motor neuron dendrites}

The precocious outgrowth of motor dendrites suggests that early interactions between these dendrites and incoming Ia afferents might influence Ia afferents en route to their distinations in the developing ventral horn. A possible hypothesis is that afferents grow without spatial order into a dense neuropil where they are in proximity to dendrites of a variety of motor pools. Specific connections with appropriate motor neurons might occur on the basis of random encounters with dendrites possessing appropriate surface markers (see Lichtman et al., 1984).

It seems likely that additional mechanisms influence the formation of specific connections in this system for several reasons. A clear-cut finding about the relationship between Ia afferents and motor dendrites emerging from this study is that some groups of afferents can grow to appropriate motor pools without any contact with motor dendrites. This is particularly apparent for afferents projecting to motor neurons that innervate axial musculature. Since these neurons do not project dendrites in gray matter, dendrites cannot influence the trajectory of innervating dorsal root afferents. Afferent axons destined for axial motor pools traverse the entire dorsoventral length of the cord prior to contacting the proximal dendrites and somata of their targets.

The situation regarding interactions between afferents and dendrites of motor neurons innervating limb musculature is more complex. These neurons project dendrites directly in the path of incoming dorsal root afferent axons. This dendritic projection is well established by E15, and these dendrites are positioned in such a way that afferents could innervate appropriate motor neurons by random encounters with dendrites of appropriate specificity. However, the predominant impression from our material is that dorsal root afferents are not guided to motor pools by these dendrites that project in their path. This idea that dendrites do not guide randomly growing afferents is supported by several observations. (1) In double-label preparations it is apparent that the major fascicles of afferents do not grow along the dendrites for significant distances. Conversely, the greatest part of the length of many dendrites is free from contact by dorsal root afferent axons. (2) Fascicles leaving the intermediate zone project straight to the motor pools and are aligned at a slightly different angle than the motor dendrites. Only a few axons make the sharp turns that would be necessary if their course were changed by contact with individual dendrites. (3) Many fibers in the afferent bundles do not defasciculate and elaborate boutons until they have reached the gray-white border, near motor neuron somata or proximal dendrites. These observations, taken together, suggest that even in limb regions, Ia afferent axons find appropriate target cells by mechanisms in addition to chance encounters with appropriate dendrites.

\section{Developing Ia afferents arborize in the region of motor neuron somata and proximal dendrites}

A significant portion of terminal branching and bouton formation of Ia axons occurs in the region of motor neuron somata and proximal dendrites. Most Ia axons remain fasciculated until they are within the vicinity of the motor neuron cell bodies, at which point they defasciculate and elaborate boutons beginning on E17. In limb regions, la afferents extend until they reach the region of motor neuron somata despite traversing an area populated by dendrites. Quantitative evidence for these observations was obtained in the C5 segment where the density of 
boutons is greater within the ventral horn areas occupied by motor neuron cell bodies (lamina IX) than in adjacent areas (lamina VII) where dendrites are projecting. Interestingly, few boutons were found along dorsally directed dendrites projecting outside lamina IX and almost none on dendrites that project in white matter. Group Ia afferents show this pattern at least through the first postnatal week. It is not clear whether the extensive bouton formation near somata and proximal dendrites reflects a preference of la axons for these structures or simply reflects the fact that fascicles of Ia axons are near the cell bodies at the time that terminal branching and bouton formation begins.

It is important to emphasize that we have studied a relatively early developmental stage and that redistribution of boutons may well occur over the course of postnatal development (Conradi and Ronnevi, 1975; see also Smolen and Raisman, 1980; Mason et al., 1990). However, this initial location of Ia terminal arbors appears to reflect their location in maturity. Previous descriptions of pairs of Ia afferents and motor neurons in cat, as well as Golgi investigations, have suggested that these afferents innervate proximal portions of the dendritic arbor (Schiebel and Schiebel, 1969; Brown, 1981). Thus, it appears that Ia afferents grow from the outset to the region of the target ncuron that they will innervate in maturity.

Motor neurons like other major CNS nutflow neurons are thought to receive different classes of afferents on different portions of the soma and dendritic arbor. Golgi investigations in cat have suggested that corticospinal and rubrospinal afferents innervate distal and dorsally directed motor neuron dendrites (Scheibel and Scheibel, 1966a,b). Whether these other afferent systems also grow directly to their final location or whether they initially overlap extensively with the Ia system cannot be determined without more detailed information about the disposition of different afferent systems on the dendritic arbors of motor neurons during development. The development of other spinal afferent systems in relation to motor neuron somata and dendrites should be possible to characterize utilizing techniques similar to the ones described here.

\section{References}

Bhide PG, Frost DO (1991) Stages of growth of hamster retinofugal axons: implications for developing axonal pathways with multiple targets. J Neurosci 11:485-504.

Brown AG (1981) Organization in the spinal cord. The anatomy and physiology of identified neurones. New York: Springer.

Chua CK, Balice-Gordon RJ, Lichtman JW (1990) Differential labeling of terminal arbors of multiple axons innervating the same target cell with DiI and a new lipophilic tracer 4-Di-16-ASP. Soc Neurosci Abstr 16:1004.

Conradi S, Ronnevi LO (1975) Spontaneous elimination of synapses on cat spinal motoneurons after birth: do half of the synapses on the cell bodies disappear? Rrain Res 92:505-510.

Davis BM, Frank E, Johnson FA, Scott SA (1989) Development of central projections of lumbosacral sensory neurons in the chick. J Comp Neurol 279:556-566.

Fitzgerald M (1987) Prenatal growth of fine-diameter primary afferents into the rat spinal cord: a transganglionic tracer study. J Comp Neurol 261:98-104.

Frank E, Westerfield M (1983) Development of sensory-motor synapses in the spinal cord of the frog. J Physiol (Lond) 343:593-610.

Godement P, Vanselow J, Thanos S, Bonhoffer F (1987) A study in developing visual systems with a new method of staining neurones and their processes in fixed tissues. Development 101:697-713.

Hongo T, Kudo N, Sasaki S, Yamashita M, Yoshida K, Ishizuka N, Mannen $H$ (1987) Trajectory of group Ia and Ib fibers from the hind-limb muscles at the L.3 and L4 segments of the spinal cord of the cat. J Comp Neurol 262:159-194.
Honig MG, Hume MI (1986) Fluorescent carbocyanine dyes allows living neurons of identifying origin to be studied in long-term cultures. J Cell Biol 103:171-178.

Jackson PC, Frank E (1987) Development of synaptic connections between muscle and sensory and motor neurons: anatomical evidence that postsynaptic dendrites grow into a preformed sensory neuropil. J Comp Neurol 255:538-547.

Kudo N, Yamada T (1987) Morphological and physiological studies of development of the monosynaptic reflex pathway in the rat lumbar spinal cord. J Physiol (Lond) 389:441-459.

Lee MT, O'Donovan MJ (1991) Organization of hindlimb muscle afferent projections to lumbosacral motoneurons in the chick embryo. J Neurosci 11:2564-2573.

Lee MT, Koebbe MJ, O'Donovan MJ (1988) The development of sensorimotor synaptic connections in the lumbosacral cord of the chick embryo. J Neurosci 8:2530-2543.

Lichtman JW, Jhaveri S, Frank E (1984) Anatomical basis of specific connections between sensory axons and motor neurons in the brachial spinal cord of the bullfrog. J Neurosci 4:1754-1763.

Mason CA, Christakos S, Catalano SM (1990) Early climbing fiber interactions with Purkinje cells in the postnatal mouse cerebellum. J Comp Neurol 297:77-90.

McHanwell S, Biscoe TJ (1981) The localization of motoneurons supplying the hindlimb muscles of the mousc. Proc R Soc London Ser B 293:477-498.

O'Leary DDM, Terashima T (1988) Cortical axons branch to multiple subcortical targets by interstitial axon budding. Implications for target recognition and "waiting periods." Neuron 1:901-910.

Réthelyi M, Szentágothai J (1973) Distribution and connections of afferent fibers in the spinal cord. In: Handbook of sensory physiology, Vol II, Somatosensory system (Iggo A, ed), pp 207-252. New York: Springer.

Romanes G (1964) The motor pools of the spinal cord. Prog Brain Res 11:93-119.

Ruit KG, Elliot JL, Osborne PA, Yan Q, Snider WD (1992) Selective dependence of mammalian dorsal root ganglion neurons on nerve growth factor during embryonic development. Neuron 8:573-587.

Sandell JH, Masland RH (1988) Photoconversion of somc fluorcscent markers to a diaminobenzidine product. J Histochem Cytochem 36: 555-559.

Scheibel ME, Scheibel AB (1966a) Terminal axonal patterns in cat spinal cord. I. The lateral corticospinal tract. Brain Res 2:333-350.

Scheibel ME, Scheibel AB (1966b) Spinal motoneurons, interneurons and Renshaw cells. A Golgi study. Arch Ital Biol 104:328-353.

Scheibel ME, Scheibel AB (1969) Terminal patters in cat spinal cord. III. Primary afferent collaterals. Brain Res 13:417-443.

Smith CL (1983) The development and postnatal organization of primary afferent projections to the rat thoracic spinal cord. J Comp Neurol 220:29-43.

Smolen A, Raisman G (1980) Synapse formation in the rat superior cervical ganglion during normal development and after deafferentation. Brain Res 181:315-323.

Snider WD, Palavali V (1990) Early axon and dendritic outgrowth of spinal accessory motor neurons studied with DiI in fixed tissues. J Comp Neurol 297:227-238.

Snider WD, Gorukanti N, Tsering C (1990) Interactions between motor neurons dendrites and dorsal root afferent axons in developing mammalian spinal cord. Soc Neurosci Abstr 16:1126.

Snider WD, Zhang L, Yusoof S, Konstantinidou A (1991) Distribution of dorsal root afferent boutons on motor neurons in developing mammalian spinal cord. Soc Neurosci Abstr 17:217.

Tyrrell S, Schroeter S, Coulter L, Tosney KW (1990) Distribution and projection pattern of motoneurons that innervate hindlimb muscles in the quail. J Comp Neurol 298:413-430.

Udin SB, Fawcett JW (1988) Formation of topographic maps. Annu Rev Neurosci 11:289-327.

Vaughn JE, Barber RP, Sims TJ (1988) Dendritic development and preferential growth into synaptogenic fields: a quantitative study of Golgi impregnated spinal motor neurons. Synapse 2:69-78.

Willis WD, Coggeshall RE (1991) Sensory mechanisms of the spinal cord. New York: Plenum.

Ziskind-Conhaim L (1990) NMDA receptors mediate poly- and monosynaptic potentials in motoneurons of rat embryos. J Neurosci 10:125-135. 